\title{
Fatores determinantes da qualidade de vida numa população de doentes com doença pulmonar obstrutiva crónica
}

Marlene Barros, * Flávio Guimarães,** Jaime Correia de Sousa***

\section{RESUMO}

Objetivos: Analisar a relação entre a Qualidade de Vida (QdV) e os seguintes fatores: género, idade, índice de massa corporal, estado civil, situação profissional, volume expiratório forçado no $10^{\circ}$ segundo, ansiedade, depressão e dispneia numa população de indivíduos com Doença Pulmonar Obstrutiva Crónica (DPOC).

Tipo de estudo: Observacional, analítico transversal.

Local: Unidade de Saúde Familiar (USF) Manuel Rocha Peixoto e USF Gualtar, Braga, Portugal.

População: Doentes com idade igual ou superior a 40 anos, diagnosticados com DPOC ou bronquite crónica, inscritos nas USF Gualtar e Manuel Rocha Peixoto.

Métodos: Os dados sociodemográficos foram recolhidos através de um formulário, composto também pelas versões portuguesas do Hospital Anxiety and Depression Scale, Medical Research Council Dyspnea Questionnaire e COPD Specific Saint George's Respiratory Questionnaire (SGRQ-C). O diagnóstico de DPOC foi confirmado através da realização de espirometria, determinando-se o volume expiratório forçado no $1 .^{\circ}$ segundo. Foi realizada uma análise bivariada entre as variáveis em estudo e a QdV. Posteriormente submeteram-se as variáveis estatisticamente significativas à regressão linear múltipla.

Resultados: Sessenta e cinco doentes foram incluídos no estudo. Na análise bivariada, a depressão, ansiedade e dispneia relacionaram-se com todos os domínios do SGRQ-C. O género e a situação profissional também mostraram uma relação significativa nos domínios Atividade, Impacto e no valor total do SGRQ-C. Posteriormente, na análise com a regressão linear múltipla, o género e a ansiedade perderam a sua influência, revelando-se, como principais determinantes, a dispneia no domínio Sintomas e, no domínio Impacto, situação profissional e dispneia no domínio Atividade e, no valor Total do SGRQ-C, a situação profissional, depressão e dispneia.

Conclusões: A situação profissional, a depressão e a dispneia deverão ser consideradas nas estratégias para melhorar a QdV nesta população de doentes com DPOC.

Palavras-chave: Doença Pulmonar Obstrutiva Crónica; Qualidade de Vida.

\section{INTRODUÇÃO}

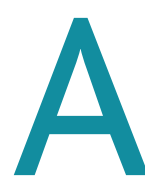

doença pulmonar obstrutiva crónica (DPOC) é uma causa importante de morbilidade crónica e mortalidade prematura, constituindo a $4 .^{\mathrm{a}}$ causa de morte em todo o mundo. ${ }^{1-9}$ Apresenta-se como a $10 .^{\text {a }}$ causa de incapacidade a nível mundial e a $12 .^{\text {a }}$ causa de anos de vida útil perdidos ajustados à idade (DALYS). ${ }^{1,10-12}$ Em Portugal, um estudo realizado no
* Médica Interna do $1 .^{\circ}$ ano de Medicina Geral e Familiar, USF do Minho, ACES Cávado I

**Médico Interno do $1 .^{\circ}$ ano de Medicina Geral e Familiar, USF Santa Clara, ACES Grande Porto IV

***Professor Associado, Instituto de Ciências da Vida e da Saúde (ICVS), Escola de Ciências da Saúde, Universidade do Minho e ICVS/3B ' s Laboratório Associado; Médico de Família, Unidade de Saúde Familiar Horizonte, Unidade Local de Saúde de Matosinhos

Trabalho realizado na Unidade de Saúde Familiar Manuel Rocha Peixoto e Unidade de Saúde Familiar Gualtar - ACES Cávado I, Braga 
âmbito da iniciativa Burden of Obstructive Lung Disease (BOLD), ${ }^{4-5}$ na região de Lisboa, determinou uma prevalência de DPOC de 14,2\% em indivíduos com idade igual ou superior a 40 anos. ${ }^{13}$

Para além da magnitude do problema, sob o ponto de vista clínico, a DPOC determina sofrimento a vários níveis, quer seja físico, psíquico ou social, levando assim a uma diminuição da Qualidade de Vida (QdV) destes doentes. $^{2}$

AQdVé atualmente uma componente essencial dos cuidados de saúde pelo que se torna importante avaliá-la., ${ }^{3,14}$ No contexto das doenças pulmonares crónicas, os dados de QdV têm-se mostrado muito úteis na avaliação do sucesso de intervenções terapêuticas, sugerindo direções a tomar na prevenção dos danos causados pela doença. ${ }^{14-17}$

Uma origem multifatorial está provavelmente na base da diminuição da QdV destes indivíduos. ${ }^{16-17}$ A presença de comorbilidades psiquiátricas tem sido relacionada com o aumento da mortalidade e diminuição do estado funcional destes indivíduos. ${ }^{3,9,14}$ Concretamente, a ansiedade e depressão têm sido muito reportadas como determinantes da QdV. ${ }^{18,21,24,26-27} \mathrm{~A}$ dispneia ${ }^{3,19-20,25-26} \mathrm{e}$ a intolerância ao exercício físico ${ }^{16-17,24-25}$ apresentam-se como duas das principais e mais comuns queixas dos doentes com DPOC, pelo que é importante perceber o seu efeito na QdV. O índice de massa corporal (IMC) ${ }^{16} \mathrm{e}$ a hipoxémia $^{21,26}$ são dois determinantes que também mostram relação com a QdV destes doentes.

Outros fatores, ainda que em menor escala ou com resultados controversos, têm sido estudados, como: idade, condições sociodemográficas e económicas, estratégias de coping e número de maços de cigarros fumados ao longo dos anos. ${ }^{14-15,19,22-24,28}$

Em Portugal existem poucos estudos sobre DPOC e $\mathrm{QdV}$, sendo importante conhecer o problema em doentes seguidos nos cuidados de saúde primários. ${ }^{29-30} \mathrm{O}$ conhecimento dos fatores que mais influenciam e determinam a QdV destes doentes permite direcionar esforços em opções de tratamento mais eficazes, racionalizando-se tempo e custos e evitando-se as consequências da morbilidade desta doença.

Assim, este estudo teve como objetivos analisar a relação entre a QdV e as seguintes variáveis: género, idade, IMC, estado civil, situação profissional, volume expiratório forçado no $1 .^{\circ}$ segundo $\left(\mathrm{FEV}_{1}\right)$, ansiedade, depressão e dispneia numa população de indivíduos com DPOC.

\section{MÉTODOS}

Foi realizado um estudo observacional descritivo, transversal e analítico nas Unidades de Saúde Familiar (USF) Gualtar e Manuel Rocha Peixoto, em Braga.

A população estudada foi constituída pelas pessoas inscritas nas USF acima referidas, com idade igual ou superior a 40 anos, diagnosticados com DPOC ou bronquite crónica (que estavam classificados no processo clínico pessoal respetivamente com o código R95 ou R79, segundo a ICPC-2, International Classification of Primary Care 2nd edition). Foram excluídos os doentes cujo contacto não foi possível, por ausência de dados administrativos, e os doentes sem domínio da língua portuguesa, escrita ou falada.

Foram enviados convites de participação no estudo a todos os doentes com DPOC ou bronquite crónica das duas USF. Posteriormente, os que não manifestaram oposição ao conteúdo da carta, num prazo de oito dias, foram contactados via telefone e convidados a comparecer nas respetivas USF para uma entrevista. Foram feitas até três tentativas de contacto telefónico em diferentes períodos do dia: manhã, início e final da tarde. A recolha dos dados ocorreu durante o mês de agosto de 2011 nas USF supracitadas. Os doentes assinaram os consentimentos informados, assegurando a confidencialidade dos dados.

Cada doente realizou uma espirometria com prova de broncodilatação, com o espirómetro MIR Spirobank G, a fim de se confirmar o diagnóstico de DPOC e obter o valor de $\mathrm{FEV}_{1}{ }^{1}{ }^{1}$ Foram ainda excluídos os doentes cujo resultado da espirometria não confirmou o diagnóstico de DPOC. Foi, assim, constituído o grupo de doentes incluídos no estudo.

Os restantes dados foram obtidos através do autopreenchimento de um questionário composto por quatro partes: Seç̧ão $\boldsymbol{A}$-Constituída pelos seguintes dados sociodemográficos: idade, género, peso e altura (para o cálculo do IMC), estado civil e situação profissional; e pelo $\mathrm{FEV}_{1}$; Secção $\boldsymbol{B}$ - Composta pela versão portuguesa da Hospital Anxiety and Depression Scale (HADS), uma escala de autopreenchimento que foi desenvolvida por Zigmond e Snaith ${ }^{31} \mathrm{e}$ validada em Portugal por Pais Ribeiro. ${ }^{32}$ A HADS tem sido largamente usada em diversas populações, inclusive em doentes com DPOC, ${ }^{21,24,26,33-35}$ estando o seu uso recomendado pela Direção-Geral de Saúde (DGS). ${ }^{36}$ É composta por duas subescalas: uma mede a ansiedade e a outra a depressão. Cada uma tem 7 itens que são respondidos numa escala de quatro pontos (0-ine- 
xistente, 3-muito grave), tendo cada subescala uma pontuação máxima de 21 pontos; Secção C - Composta pela versão portuguesa do COPD-Specific Saint George's Respiratory Questionnaire (SGRQ-C), desenvolvido a partir do St. George's Respiratory Questionnaire (SGRQ). ${ }^{37-39} \mathrm{O}$ SGRQ é o instrumento gold standard na medição da QdV em doentes respiratórios crónicos, mas não é específico para a DPOC. ${ }^{14-21,23-28}$ O SGRQ-C é uma versão mais curta e cujos valores podem ser facilmente convertidos em valores correspondentes da versão original, permitindo assim a comparação com estudos que a utilizem. Já validado e traduzido para português, ${ }^{40}$ o SGRQ-C foi desenvolvido utilizando apenas dados sobre a DPOC. É composto por três domínios englobados em duas partes: Parte 1 (questões 1-7), respeitante à componente dos Sintomas, aborda a frequência dos sintomas respiratórios; e a Parte 2 (questões 8-14), engloba as componentes Atividade e Impacto, aborda o estado atual do paciente. O domínio Atividade mede os distúrbios da atividade física diária, enquanto o domínio Impacto abrange os distúrbios da função psicossocial. Cada resposta tem uma ponderação que varia entre 0 e 100 . Cada domínio pode ser calculado separadamente, bem como a pontuação Total. O resultado varia entre 0 e $100 \%$, sendo que quanto maior o valor, pior a QdV. Foi desenhado para um autopreenchimento supervisionado e a administração por telefone também se encontra validada; ${ }^{41-42}$ Secção $D$ - Composta pela versão portuguesa do Medical Research Council Dyspnea Questionnaire (MRCDQ) numa escala de 5 graus, sendo os graus 0 e 1 os de menor sensação de dispneia e o grau 4 o de maior. ${ }^{43}$ Esta é uma escala que integra a revisão GOLD 2013 e o seu uso está aconselhado pela DGS, ${ }^{36}$ sendo largamente usada em diversos estudos internacionais. ${ }^{20,26,33}$ No caso de doentes analfabetos, o formulário foi preenchido pelo investigador.

A análise estatística dos dados foi feita com o programa IBM SPSS Statisticsv.19, recorrendo-se ao Teste- $t$ para amostras independentes e ao Teste do Qui-quadrado para comparação de médias e proporções e medindo-se a dimensão do efeito através do cálculo do $d d e$ Cohen e odds ratio, respetivamente. Para a análise bivariada entre as variáveis em estudo e a QdV utilizaram-se o Teste- $t$ para amostras independentes e a Correlação de Pearson. Posteriormente submeteram-se as variáveis com diferenças estatisticamente significativas e significativamente correlacionadas à regressão linear múltipla. $\mathrm{O}$ nível de significância adotado foi de $0,05 . .^{44-46}$

\section{RESULTADOS}

Observou-se que 350 doentes estavam codificados com DPOC (R95) ou bronquite crónica (R79), constituindo a população do estudo. Desses, $55(15,7 \%)$ preenchiam os critérios de exclusão, $125(35,4 \%)$ recusaram o convite, 54 (15,4\%) não atenderam os telefonemas e 15 $(4,3 \%)$ faltaram ao encontro agendado. Dos 101 doentes participantes no estudo, 36 foram posteriormente excluídos devido à não confirmação do diagnóstico de DPOC na espirometria com prova de broncodilatação. Como resultado, o grupo de doentes incluídos no estudo foi de 65 pessoas (figura 1).

De forma a avaliar a representatividade da amostra em estudo, analisou-se a relação entre os doentes participantes no estudo e os não participantes, não se verificando diferenças estatisticamente significativas, relativamente ao género e à idade $(p=0,053 \mathrm{e} p=0,106)$. No entanto, analisando a relação entre os doentes incluídos no estudo e os excluídos e não participantes verificou-se existir uma diferença estatisticamente significativa para o género ( $p=0,020 ; d=1,969)$ (tabela I).

A dimensão do efeito foi calculada como forma de complementar e melhorar o teste estatístico, uma vez que o seu valor é independente da dimensão amostral. Com base nos critérios de Cohen, verificou-se que, no presente estudo, todos os resultados estatisticamente significativos foram associados a grandes dimensões do efeito.

\section{Caracterização sociodemográfica e clínica da amostra}

Os doentes incluídos no estudo estão caracterizados na tabela II, de acordo com as características sociodemográficas e clínicas. A amostra era constituída por 45 elementos do género masculino $(69,2 \%)$ e 20 elementos do género feminino (30,8\%), com média de idades de 67 $\pm 10,2$ anos e média de IMC de $27 \pm 4,5 \mathrm{Kg} / \mathrm{m}^{2} .80 \%$ dos doentes eram casados e $86,2 \%$ não trabalhavam.

No que respeita à sensação de dispneia, 33 doentes $(50,8 \%)$ encontram-se entre os graus de menor gravidade (graus 0 e 1), enquanto 32 doentes $(49,2 \%)$ se encontram nos graus 2 ou superiores. A média de $\mathrm{FEV}_{1}$ obtido foi de $74 \pm 21,2 \%$ do previsto. Quanto à depressão e ansiedade, a média das respostas obtidas foi de $7 \pm 4,3$ e 8 $\pm 4,2$, respetivamente.

No SGRQ-C, $53 \pm 19,9 \%$ foi a média obtida no domínio Sintomas, $52 \pm 24,1 \%$ no domínio Atividade, $36 \pm$ $24,1 \%$ no domínio Impacto e $44 \pm 20,9 \%$ foi a média do valor Total. 


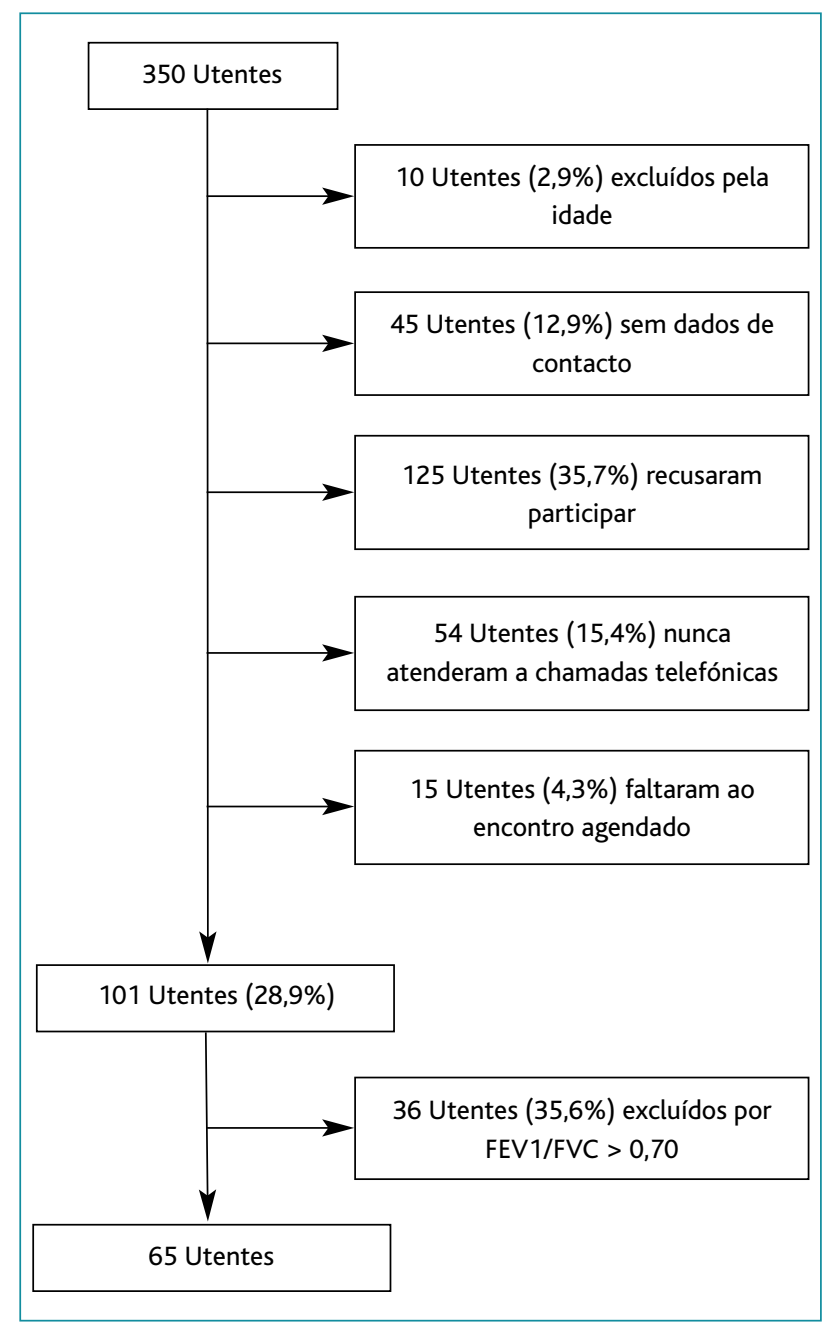

Figura 1. Constituição da amostra a partir da população em estudo.

\section{Identificação dos determinantes da QdV}

$\mathrm{Na}$ análise da relação bivariada entre as variáveis estudadas e a QdV procedeu-se ao teste- $t$ para amostras independentes (tabela III) e a correlações de Pearson (tabela IV). Posteriormente, as variáveis mais relacionadas com o resultado foram incluídas na regressão linear múltipla, identificando-se assim as que melhor predizem os diferentes domínios da QdV (tabela V).

De acordo com as tabelas III e IV, verifica-se que, no domínio Sintomas, as variáveis que se relacionaram de forma significativa foram a dispneia, depressão e ansiedade, tendo sido estas as variáveis avaliadas na regressão linear múltipla (tabela $\mathrm{V}$ ). Na avaliação simultânea das variáveis, a depressão e a ansiedade perderam a sua influência, ficando apenas a dispneia $(p<0,001 ; \beta=0,317)$ como significativamente influente neste domínio de QdV, explicando 32,7\% da sua variabilidade.

Quanto aos domínios Atividade, Impacto e valor Total do SGRQ-C foram as variáveis independentes género, situação profissional, dispneia, depressão e ansiedade que se relacionaram significativamente, mas de forma isolada, com a QdV. No entanto, quando se avaliou a influência destes fatores em simultâneo, verificou-se que, no domínio Atividade, as variáveis situação profissional $(p=0,007 ; \beta=0,263)$ e dispneia $(p<0,001 ; \beta=0,433)$ foram os melhores preditores, explicando $53,1 \%$ da variabilidade deste domínio.

No domínio Impacto, $53,1 \%$ da variabilidade é explicada apenas pela dispneia ( $p<0,001 ;=0,443)$.

A situação profissional ( $p=0,021 ; \beta=0,204)$, a depressão $(p=0,026 ; \beta=0,259)$ e a dispneia $(p<0,001 ; \beta=$ 0,480 ) mostraram também a sua influência no valor Total do SGRQ-C, devendo-se a estes fatores $60,7 \%$ da sua variabilidade.

Após a conversão dos valores do SGRQ-C para serem comparáveis com os valores do $\mathrm{SGRQ}^{40}$ verificaram-se os mesmos resultados.

\section{DISCUSSÃO}

Os resultados deste estudo demonstraram que a situação profissional, a depressão e a dispneia foram os principais fatores determinantes da QdV nesta população de doentes com DPOC.

Os doentes incluídos no estudo eram maioritariamente do sexo masculino $(69,2 \%)$ e a média de idades foi de 67 anos. Estes dados são apoiados pelo relatório do observatório nacional para as doenças respiratórias, de 2009, que refere que a prevalência de DPOC em Portugal, apesar de subestimada, é superior nos homens e em idades mais avançadas. ${ }^{10}$

As mulheres demonstraram valores mais elevados no SGRQ-C, com uma diferença estatisticamente significativa relativamente aos homens na maioria dos domínios e no valor Total, implicando pior QdV. Apesar de a diferença estatística não se ter verificado no domínio Sintomas, as mulheres tiveram mais cinco pontos do que os homens, sendo que quatro pontos é o limiar para se considerar esta diferença clinicamente significativa. ${ }^{41}$ Este resultado é semelhante ao obtido por De Torres et $\mathrm{al}^{46}$ num estudo realizado com 53 homens e 53 mulheres, onde se concluiu que as mulheres obtiveram piores resultados 


\begin{tabular}{|c|c|c|c|c|c|c|}
\hline & \multicolumn{2}{|c|}{$\begin{array}{l}\text { Participantes } \\
(\mathrm{n}=101)\end{array}$} & $\begin{array}{l}\text { Não participantes } \\
\quad(n=249)\end{array}$ & $\begin{array}{c}\text { Teste } \\
\text { estatístico }\end{array}$ & $P$ & $\begin{array}{l}\text { Odds ratio/ } \\
\text { /d de Cohen }\end{array}$ \\
\hline \multicolumn{7}{|l|}{ Género, n(\%) } \\
\hline Masculino & \multicolumn{2}{|c|}{$65(18,6)$} & $132(37,7)$ & $x^{2}(1)-3758$ & 0053 & \\
\hline Feminino & \multicolumn{2}{|c|}{$36(10,3)$} & $117(33,4)$ & 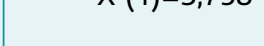 & כבנט, & 1,0 \\
\hline Idade, & \multirow{2}{*}{\multicolumn{2}{|c|}{$65,9(10,7)$}} & & & & \\
\hline \multirow{2}{*}{$\begin{array}{l}\text { Média (Desvio } \\
\text { Padrão) }\end{array}$} & & & $68,4(15,8)$ & $t(271,336)=1,622$ & 0,106 & 0,185 \\
\hline & $\begin{array}{l}\text { Incluídos } \\
(\mathrm{n}=65)\end{array}$ & $\begin{array}{l}\text { Excluídos } \\
(\mathrm{n}=36)\end{array}$ & & & & \\
\hline \multicolumn{7}{|l|}{ Género, n(\%) } \\
\hline Masculino & $45(44,6)$ & $20(19,8)$ & & $X 2(1)=1,889$ & 0,169 & 1,8 \\
\hline Feminino & $20(19,8)$ & $16(15,8)$ & & & & \\
\hline \multicolumn{7}{|l|}{ Idade, } \\
\hline \multirow{2}{*}{$\begin{array}{l}\text { Média (Desvio } \\
\text { Padrão) }\end{array}$} & $66,9(10,2)$ & $64,4(11,4)$ & & $t(99)=-1,145$ & 0,255 & 0,231 \\
\hline & $\begin{array}{l}\text { Incluídos } \\
(\mathrm{n}=65)\end{array}$ & \multicolumn{2}{|c|}{$\begin{array}{l}\text { Excluídos + Não participantes } \\
\qquad(\mathbf{n}=\mathbf{2 8 5})\end{array}$} & & & \\
\hline \multicolumn{7}{|l|}{ Género, n(\%) } \\
\hline Masculino & $45(12,9)$ & & $152(43,4)$ & $X 2(1)=5,437$ & 0,020 & 1,969 \\
\hline Feminino & $20(5,7)$ & & $133(38)$ & & & \\
\hline \multicolumn{7}{|l|}{ Idade, } \\
\hline $\begin{array}{l}\text { Média (Desvio } \\
\text { Padrão) }\end{array}$ & $66,9(10,2)$ & & $67,9(15,4)$ & $t(138,725)=-0,615$ & 0,540 & 0,077 \\
\hline
\end{tabular}

em todos os domínios e no valor total do SGRQ. No entanto, os estudos de Ketelaars et $\mathrm{al}^{15} \mathrm{e}$ de Stahl et $\mathrm{al},{ }^{28}$ usando o mesmo questionário, não encontraram diferenças estaticamente significativas para o género. Um estudo realizado por Ferrer et al ${ }^{42}$ no âmbito do projeto IBERPOC (Epidemiological Study of Chronic Obstructive Pulmonary Disease in Spain), não demonstrou diferenças estatisticamente significativas no SGRQ entre homens e mulheres com DPOC, com exceção do domínio Sintomas.

Estes autores avaliaram também as diferenças entre género numa população saudável e concluíram que as mulheres apresentavam menos sintomas respiratórios, mas piores índices de atividade, indicando que as diferenças no valor do SGRQ são parcialmente independentes da doença respiratória. No presente estudo, apesar de veri- ficada a diferença por género, este perdeu influência ao ser avaliado simultaneamente com outros fatores. Também Renwick e Connolly, ${ }^{23}$ num estudo efetuado com 190 doentes, submeteram o género à avaliação simultânea com outros preditores, não se verificando a influência deste sobre a QdV. Face a estes resultados, pensa-se que, na amostra em estudo, as diferenças observadas podem não ter sido verdadeiramente devidas ao género mas a outros preditores, como a depressão, identificados na literatura como mais frequentes no género feminino. ${ }^{27,42}$

Neste estudo foram ainda avaliados outros dados sociodemográficos, como a idade, o estado civil e a situação profissional. Destes, apenas a situação profissional mostrou influenciar os domínios Atividade e valor Total, ainda que em menor escala quando comparada com a 
TABELA II. Caracterização sociodemográfica e clínica da amostra $(n=65)$

\begin{tabular}{l|c} 
Variáveis & $\mathbf{n}(\%)$ \\
\hline Género & \\
Masculino & $45(69,2)$ \\
Feminino & $20(30,8)$
\end{tabular}

\begin{tabular}{l|l}
\hline Estado civil & \\
Casado & $52(80)$ \\
Não casado & $13(20)$
\end{tabular}

Situação profissional

Trabalha

$9(13,8)$

Não trabalha

$56(86,2)$

Dispneia

Graus 0-1

$33(50,8)$

Graus $\geq 2$

$32(49,2)$

Média (Desvio padrão)

Idade (anos) $67(10,2)$

IMC $\left(\mathrm{Kg} / \mathrm{m}^{2}\right) \quad 27(4,5)$

$\mathrm{FEV}_{1}$ (\% do previsto) $\quad 74(21,2)$

\begin{tabular}{l|l}
\hline Depressão & $7(4,3)$
\end{tabular}

Ansiedade

$8(4,2)$

SGRQ-C

Sintomas (\%)

$53(19,9)$

Atividade (\%)

$52(24,1)$

Impacto (\%)

$36(24,1)$

Total (\%)

$44(20,9)$

depressão e/ou a dispneia, também preditores destes domínios. Sendo a média de idades dos doentes incluídos no estudo de 67 anos, a maioria (86,6\%) não trabalhava, encontrando-se possivelmente reformada.

Ketelaars et $\mathrm{al}^{15}$ estudaram os determinantes da QdV em 126 pacientes dos 40 aos 80 anos com DPOC grave. Concluíram que a idade não se correlacionava com a $\mathrm{QdV}$, provavelmente porque esses pacientes se ajustariam às perdas psicossociais e, como eram na maioria idosos, teriam desenvolvido novas atitudes e adequado o seu estilo de vida às limitações decorrentes da doença, encarando-as como consequência natural da idade. Este facto é apoiado pelos resultados do presente estudo, onde também não se obteve qualquer relação entre a idade e a QdV. Contudo, Stahl et $\mathrm{al}^{28}$ demonstraram, num estudo feito na Suécia com 168 indivíduos com DPOC, que a QdV diminuía com o aumento da idade. Ferrari et al, ${ }^{47}$ num estudo com 60 homens e 30 mulheres, procuraram identificar os principais preditores da QdV de acordo com o género e verificaram que a idade era determinante em ambos os géneros. Verifica-se, assim, que os dados sociodemográficos não têm obtido resultados consensuais, sendo necessários estudos que contemplem a uniformização das metodologias e populações para que se apure a sua verdadeira influência na QdV.

Quanto ao IMC, neste estudo, não se verificou qualquer relação com a QdV. O mesmo foi verificado por Tsukino et al, ${ }^{22}$ quando avaliaram a relação entre o peso corporal e a QdV (avaliada com o Nottingham Health Profile e Chronic Respiratory Questionnaire) em 132 doentes. Por outro lado, Dourado et $\mathrm{al}^{16}$ avaliaram 21 doentes com DPOC moderada a grave e verificaram a existência de correlações negativas entre o IMC e os domínios Impacto e Sintomas e valor Total do SGRQ.

Os mesmos autores avaliaram também o $\mathrm{FEV}_{1}$ e concluíram que, apesar de negativamente correlacionado com o domínio Impacto e valor Total do SGRQ, não se demonstrou determinante para a QdV na avaliação simultânea com outros fatores. $\mathrm{OFEV}_{1}$ foi usado neste estudo como variável independente contínua e não demonstrou qualquer efeito estatisticamente significativo na QdV. Este é também um fator bastante avaliado nos mais diversos estudos, verificando-se resultados controversos. Okubadejo et $\mathrm{al}^{21}$, num estudo efetuado a 41 indivíduos com DPOC e utilizando o SGRQ, não encontraram correlação significativa entre o $\mathrm{FEV}_{1}$ e a QdV. Estes resultados são ainda apoiados pelos estudos de Ketelaars et al, ${ }^{15}$ Engström et $\mathrm{al}^{24} \mathrm{e}$ Sant'Anna et al. ${ }^{25}$ Renwick e Connolly avaliaram a influência do $\mathrm{FEV}_{1}$ na QdV de 190 doentes, usando o SGRQ, e verificaram uma correlação significativa na análise bivariada mas uma correlação fraca na regressão linear múltipla. ${ }^{23}$

No entanto, existem estudos que categorizam o $\mathrm{FEV}_{1}$ comparando a QdV nos diferentes estadios da DPOC. ${ }^{1}$ Stahl e os seus colegas verificaram que a severidade da DPOC (e indiretamente o $\mathrm{FEV}_{1}$ ) influenciou os valores do SGRQ, com diferença estatisticamente significativa para o valor Total. ${ }^{28}$ Num estudo de coorte espanhol, Ferrer et al demonstraram que os valores do SGRQ aumentavam com o aumento do estádio da DPOC em doentes com e sem comorbilidades. ${ }^{19}$ Também os estudos efetuados por Hajiro e a sua equipa apoiam estes resultados. ${ }^{20,26}$ 


\begin{tabular}{|c|c|c|c|c|c|}
\hline \multirow[b]{2}{*}{ SGRQ-C (\%) } & \multicolumn{2}{|c|}{ Género $(n=65)$} & \multirow[b]{2}{*}{$t(63)$} & \multirow[b]{2}{*}{$\mathbf{P}$} & \multirow[b]{2}{*}{ d de Cohen } \\
\hline & $\begin{array}{l}\text { Masculino } \\
\text { M (DP) }\end{array}$ & $\begin{array}{l}\text { Feminino } \\
M(D P)\end{array}$ & & & \\
\hline Sintomas & $51,2(20,8)$ & $56,9(17,7)$ & 1,070 & 0,145 & 0,295 \\
\hline Atividade & $47,2(24,8)$ & $63,7(18,5)$ & 2,652 & 0,005 & 0,754 \\
\hline Impacto & $31,5(24,3)$ & $45,3(21,5)$ & 2,186 & 0,017 & 0,602 \\
\hline \multirow[t]{3}{*}{ Total } & $39,8(21,5)$ & $53(16,5)$ & 2,437 & 0,009 & 0,689 \\
\hline & \multicolumn{2}{|c|}{ Estado civil $(n=65)$} & & & \\
\hline & $\begin{array}{l}\text { Casados } \\
M(D P)\end{array}$ & $\begin{array}{c}\text { Não casados } \\
\text { M (DP) }\end{array}$ & & & \\
\hline Sintomas & $53,8(21,0)$ & $49,5(14,9)$ & 0,687 & 0,248 & 0,236 \\
\hline Atividade & $52,0(25,0)$ & $53,6(21,1)$ & $-0,219$ & 0,414 & 0,069 \\
\hline Impacto & $34,4(23,7)$ & $41,3(26,2)$ & $-0,918$ & 0,181 & 0,276 \\
\hline \multirow[t]{3}{*}{ Total } & $43,2(21,1)$ & $46,5(20,4)$ & $-0,508$ & 0,307 & 0,159 \\
\hline & \multicolumn{2}{|c|}{ Situação profissional $(n=65)$} & & & \\
\hline & $\begin{array}{c}\text { Trabalhadores } \\
\text { M (DP) }\end{array}$ & $\begin{array}{c}\text { Não trabalhadores } \\
\text { M (DP) }\end{array}$ & & & \\
\hline Sintomas & $50,5(25,5)$ & $53,3(19,1)$ & $-0,390$ & 0,349 & 0,124 \\
\hline Atividade & $30,9(21,8)$ & $55,7(22,9)$ & $-3,044$ & 0,002 & 1,111 \\
\hline Impacto & $18,3(12,7)$ & $38,6(24,4)$ & $-2,434$ & 0,009 & 1,043 \\
\hline \multirow[t]{3}{*}{ Total } & $27,8(16,4)$ & $46,5(20,5)$ & $-2,592$ & 0,006 & 1,009 \\
\hline & \multicolumn{2}{|c|}{ Dispneia $(n=65)$} & & & \\
\hline & $\begin{array}{c}\text { Graus } 0-1 \\
\text { M (DP) }\end{array}$ & $\begin{array}{c}\text { Graus } \geq 2 \\
M(D P)\end{array}$ & & & \\
\hline Sintomas & $43,9(19,2)$ & $62,3(16,1)$ & $-4,183$ & $<0,001$ & 1,039 \\
\hline Atividade & $38,6(21,3)$ & $66,4(18,2)$ & $-5,661$ & $<0,001$ & 1,405 \\
\hline Impacto & $22,2(18,0)$ & $49,8(21,6)$ & $-5,592$ & $<0,001$ & 1,386 \\
\hline Total & $31(16,4)$ & $57,1(16,3)$ & $-6,417$ & $<0,001$ & 1,596 \\
\hline
\end{tabular}

M - média; DP - desvio padrão.

Verificamos, então, que na maioria dos estudos em que o $\mathrm{FEV}_{1}$ é utilizado como variável quantitativa contínua, a relação com a QdV é fraca ou ausente, como verificado no presente trabalho. No entanto, quando avaliam a gravidade da doença, e indiretamente o $\mathrm{FEV}_{1}$, este relacionase com a QdV. É, assim, necessário ter cuidado ao tentar predizer a QdV com base nos resultados espirométricos.

Quanto à sensação de dispneia, avaliada no presente estudo com o MRCDQ, mostrou-se como o preditor mais forte em todos os domínios do SGRQ-C. Hajiro et $\mathrm{al}^{20}$ concluíram, num estudo realizado em 1999, que a sensação de dispneia, avaliada pelo MRCDQ, é uma determinante para a QdV mais importante do que a gravidade da doença. Os mesmos autores, em 2001, estudaram 218 indivíduos com DPOC estável e demonstraram a forte influência da dispneia em todos os domínios do SGRQ. ${ }^{26}$ Estas conclusões são ainda apoiadas pelo estudo feito por Sant'Anna et $\mathrm{al}^{25}$ em 69 indivíduos com DPOC, onde se demonstrou a correlação significativa entre a dispneia e a QdV. Também Ferrari e os seus colaboradores utilizaram o MRCDQ para avaliarem a dispneia e verificaram a sua forte influência na QdV em ambos os géneros. ${ }^{47}$

A dispneia tem vindo, assim, a ser consensualmente confirmada como determinante da QdV e, sendo também um importante fator de prognóstico, deve ser considerada na avaliação da QdV destes doentes.

As comorbilidades psiquiátricas têm sido fortemente reportadas como determinantes da QdV. No presente trabalho, tanto a depressão como a ansiedade demonstraram forte relação com o SGRQ-C quando avaliadas separadamente. No entanto, apenas a depressão, na avaliação pela regressão linear múltipla, manteve a sua influência no valor Total do SGRQ-C, revelando-se, a par da dispneia e da situação profissional, como uma determinante para a QdV. Este resultado é apoiado por Engström et $\mathrm{al}^{24}$ que, num estudo efetuado com 68 doentes, confirmou, na análise bivariada, a correlação entre a ansiedade e depressão e o SGRQ, mas na análise de regressão múltipla apenas a depressão se mostrou como preditiva da QdV. Ng et al, ${ }^{34}$ 
TABELA VII. Resultados das correlações de Pearson entre os diferentes domínios e o valor total do SGRQ-C e as variáveis avaliadas nos 65 doentes com DPOC analisados

\begin{tabular}{l|r|r|r|r}
\multirow{2}{*}{ Variáveis } & \multicolumn{4}{|c}{ SGRQ-C ( $\mathbf{n}=65)$} \\
\cline { 2 - 5 } Idade & Sintomas & \multicolumn{1}{c}{ Atividade } & \multicolumn{1}{c}{ Impacto } & \multicolumn{1}{c}{ Total } \\
\hline IMC & $r=-0,046$ & $r=0,237$ & $r=0,088$ & $r=0,129$ \\
\hline Depressão & $r=0,478^{* * *}$ & $r=0,523^{* * *}$ & $r=0,561^{* * *}$ & $r=0,598^{* * *}$ \\
Ansiedade & $r=0,377^{* *}$ & $r=0,363^{* *}$ & $r=0,451^{* * *}$ & $r=0,459^{* * *}$ \\
\hline FEV & $r=-0,100$ & $r=0,033$ & $r=-0,015$ & $r=-0,014$
\end{tabular}

${ }^{*} \mathrm{p}<0,05 ;{ }^{* *} \mathrm{p}<0,01 ;{ }^{* * *} \mathrm{p}<0,001$

num estudo de coorte com 376 doentes, demonstraram, através da análise multivariada, a forte associação entre a depressão e o SGRQ, independentemente da severidade da DPOC, comorbilidades e variáveis socioeconómicas, psicossociais e comportamentais. Hajiro et $\mathrm{al}^{26}$ avaliaram também a influência dos sintomas psicológicos na QdV, confirmando a importância da depressão e ansiedade no bem-estar dos doentes. Num estudo realizado em 2006, Di Marco et $\mathrm{al}^{27}$ documentaram uma correlação estatisticamente significativa entre a ansiedade e depressão e o SGRQ. Outros estudos, usando as mesmas escalas de medida, apoiam estes resultados, como é o caso da investigação realizada por Okubadejo et al. ${ }^{24}$

Outras variáveis poderiam ter sido testadas, nomeadamente a da classe social ou o do teste de caminhada de 6 minutos. No entanto, e tendo em conta o número de doentes que compunham a amostra, procurou-se, por um lado, testar variáveis já citadas na literatura como potenciais determinantes para a QdV e, por outro, não incluir variáveis que fossem obtidas de forma invasiva ou agressiva para os participantes e que limitasse a sua participação ou que não fossem passíveis de serem medidas devido a fatores externos aos investigadores. A classe social é exemplo de uma variável que, apesar de ser facilmente obtida, não foi incluída pois tem vindo a ser nega- da como determinante da QdV. ${ }^{15,28}$ Por outro lado, aconselha-se a inclusão do teste de caminhada de 6 minutos em futuras investigações, pois tem sido frequentemente apontado como determinante da QdV em diversos estudos. ${ }^{15-16,24-25}$

Este estudo apresenta algumas limitações. Apesar da baixa taxa de participação (28,9\%), os 101 doentes não demonstraram diferenças estatisticamente significativas relativamente à restante população quanto ao género e idade. No entanto, após a exclusão de 36 pessoas, por não confirmação do diagnóstico de DPOC, verificou-se diferença estatística no género (tabela I). Isto pode ser explicado pela exclusão maioritariamente de mulheres, onde não se confirmou o diagnóstico, relativamente à população masculina onde a DPOC é mais frequente. $\mathrm{O}$ principal motivo da não participação no estudo foi a recusa direta dos doentes, facto que pode ser parcialmente explicado devido à necessidade de deslocação à USF. A impossibilidade de contactar algumas pessoas constituiu o segundo motivo de não participação no estudo e incluiu os que apresentavam dados de contacto incompletos, errados ou desatualizados, bem como aqueles que não responderam às três tentativas de contacto telefónico. A seleção dos utentes com base nos registos dos médicos assistentes e a utilização de uma amostra por conveniência constituem também vieses de seleção.

\begin{tabular}{|c|c|c|c|c|}
\hline \multirow{2}{*}{$\begin{array}{l}\text { Variáveis } \\
\text { independentes }\end{array}$} & \multicolumn{4}{|c|}{ SGRQ-C $(n=65)$} \\
\hline & Sintomas ${ }^{a}$ & Atividade $^{\mathrm{b}}$ & Impacto $^{c}$ & Total $^{\mathrm{d}}$ \\
\hline Género & $x$ & $\beta=-0,105$ & $\beta=-0,001$ & $\beta=-0,008$ \\
\hline Situação profissional & $x$ & $\beta=0,263^{*}$ & $\beta=0,182$ & $\beta=0,204^{*}$ \\
\hline Depressão & $\beta=0,277$ & $\beta=0,223$ & $\beta=0,229$ & $\beta=0,259^{*}$ \\
\hline Ansiedade & $\beta=0,133$ & $\beta=0,076$ & $\beta=0,195$ & $\beta=0,173$ \\
\hline Dispneia & $\beta=0,317^{*}$ & $\beta=0,433 * * *$ & $\beta=0,443^{* * *}$ & $\beta=0,480 * * *$ \\
\hline
\end{tabular}

$x$ Não testado; ${ }^{*} \mathrm{p}<0,05 ;{ }^{* *} \mathrm{p}<0,01 ;{ }^{* * *} \mathrm{p}<0,001$

$a-R^{2}=0,327 ; R^{2}$ ajustado $=0,293 ; F(3,60)=9,699 ; p<0,001$

$b-R^{2}=0,531 ; R^{2}$ ajustado $=0,490 ; F(5,58)=13,119 ; p<0,001$

$c-R^{2}=0,531 ; R^{2}$ ajustado $=0,490 ; F(5,58)=13,120 ; p<0,001$

$d-R^{2}=0,607 ; R^{2}$ ajustado $=0,573 ; F(5,58)=17,940 ; p<0,001$ 
A ausência de validação para a população portuguesa dos questionários SGRQ e MRCDQ apresenta-se como outra limitação, condicionando a sua aplicação e inferência dos resultados na população em estudo. No entanto, utilizaram-se traduções oficiais de ambos para minimizar essa limitação. ${ }^{39,43}$ Além disso, tanto o SGRQ como o MRCDQ são recomendados pela DGS na avaliação de sintomas e QdV em doentes com DPOC. ${ }^{36}$ No que respeita ao SGRQ, indicado como instrumento gold standard na medição da QdV em doentes respiratórios crónicos, procurou-se utilizar um instrumento ainda mais específico, aplicando-se a versão desenvolvida para indivíduos com DPOC. .0-41 $^{40}$

A espirometria foi o método usado na confirmação do diagnóstico de DPOC. Segundo o GOLD, a DPOC é confirmada quando a razão entre o $\mathrm{FEV}_{1}$ e a capacidade vital forçada é inferior a 0.7 pós-broncodilatação, independentemente da idade, ${ }^{1}$ tendo sido este o critério utilizado neste estudo. No entanto, não são considerados os limites inferiores da normalidade para a idade e que variam entre adultos jovens e idosos. Num estudo realizado por Güder et $\mathrm{al}^{47}$ concluiu-se que os critérios GOLD sobre diagnosticam a DPOC na população idosa, enquanto os limites inferiores da normalidade identificam um menor número de casos. Estes autores propõem, assim, a incorporação dos limites inferiores da normalidade para um diagnóstico mais preciso de DPOC.

A não inclusão de comorbilidades, nomeadamente cardiovasculares, diabetes, osteoporose, anemia ou disfunção músculoesquelética, que afetam principalmente doentes em estádio avançado e cuja influência na QdV foi já reportada, ${ }^{19}$ pode também influenciar os resultados. No entanto, o facto de os doentes terem que se deslocar às USF para a recolha dos dados acabou por incluir na amostra aqueles com doença mais estável.

A dimensão amostral desta investigação limitou o número de variáveis a incluir no modelo de regressão linear múltipla, pelo que, à semelhança de outros estudos, se optou por utilizar previamente uma análise bivariada. ${ }^{15-16,23}$ No entanto, é necessário ter em conta que, por vezes, variáveis com correlação bivariada não significativa adquirem significância em modelos de regressão múltipla, sendo que o inverso foi verificado neste estudo (o género e a ansiedade perderam a significância demonstrada anteriormente). Por outro lado, as dimensões do efeito calculadas (d de Cohen, correlação e odds ratio) refletem a magnitude do resultado obtido com o teste estatístico, independentemente do tamanho da amostra. Estas me- didas ajudam, assim, a determinar se foi encontrado um efeito clinicamente significativo, ajudam a determinar o tamanho da amostra em estudos futuros e facilitam a comparação entre investigações científicas. ${ }^{44}$

Os vieses descritos limitam a extrapolação dos resultados obtidos. Além disso, o estudo foi realizado numa população de uma zona geográfica específica de Portugal pelo que não é possível generalizar os resultados à população portuguesa.

Em conclusão, verificou-se, através dos resultados deste estudo, que a depressão, a situação profissional e a dispneia explicam cerca de $60 \%$ da variabilidade do valor Total do SGRQ-C. Este estudo demonstrou, assim, que estas variáveis deverão ser consideradas nas estratégias para melhorar a QdV nesta população de doentes com DPOC e que existem outras variáveis que influenciam a QdV, explicando os restantes $40 \%$ de variabilidade.

No futuro recomenda-se a realização de estudos que validem os instrumentos de medida e a realização de investigações com uniformização das populações, de técnicas de medição e de tratamento de dados para que se possa determinar a influência de novos fatores, e de outros ainda controversos, no sentido de se encontrar o melhor modelo a predizer a QdV nos doentes com DPOC.

\section{AGRADECIMENTOS}

Os autores agradecem à USF Gualtar, na pessoa de sua coordenadora,Dr. ${ }^{a}$ Márcia Osório Millet, e à USF Manuel Rocha Peixoto, na pessoa de sua coor-

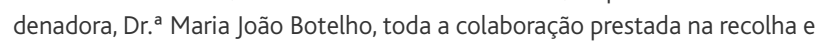
fornecimento dos dados; ao Dr. Luís Silva, por toda a ajuda na elaboração do trabalho; ao Professor Patrício Costa, pelo apoio e orientação no trabalho estatístico; ao Dr. Manuel Montenegro, pela revisão das referências bibliográficas; e também ao José Miguel Lopes Moreira, à Sílvia Castro e à técnica cardiopulmonar Raquel Barros, por tornarem possível a realização das espirometrias.

\section{REFERÊNCIAS BIBLIOGRÁFICAS}

1. Global Initiative for Chronic Obstructive Lung Disease. Global strategy for the diagnosis, management, and prevention of chronic obstructive pulmonary disease: update 2013. GOLDCOPD; 2013 [cited 2013 May 2]. Available from: http://www.goldcopd.org

2. Mannino DM.COPD: epidemiology, prevalence, morbidity and mortality, and disease heterogeneity. Chest. 2002;121(5 Suppl):121S-126S.

3. Belfer $\mathrm{MH}$, Reardon JZ. Improving exercise tolerance and quality of life in patients with chronic obstructive pulmonary disease. J Am Osteopath Assoc. 2009;109(5):268-78.

4. Buist AS, Vollmer WM, Sullivan SD, Weiss KB, Lee TA, Menezes AM, et al. The Burden of Obstructive Lung Disease Initiative (BOLD): rationale and design. COPD. 2005;2(2):277-83.

5. Buist AS, McBurnie MA, Vollmer WM, Gillespie S, Burney P, Mannino DM, et al. International variation in the prevalence of COPD (The BOLD Study): a 
population-based prevalence study. Lancet. 2007;370(9589):741-50.

6. Cardoso J, Ferreira JR, Almeida J, Santos JM, Rodrigues F, Matos MJ. Prevalence of chronic obstructive pulmonary disease (COPD) in Portugal. Am J Respir Crit Care Med. 2003;167:A110.

7. World Health Organization. World health statistics 2008. Geneva: WHO; 2009 [cited 2011 May 4].Available from: http://www.who.int/gho/ncd/mortality_morbidity/chronic_respiratory_diseases/en/index.html

8. Rabe KF, Hurd S, Anzueto A, Barnes PJ, Buist SA, Calverley P, et al. Global strategy for the diagnosis, management, and prevention of chronic obstructive pulmonary disease: GOLD executive summary. Am J Respir Crit Care Med. 2007;176(6):532-55.

9. Putman-Casdorph H, McCrone S. Chronic obstructive pulmonary disease, anxiety, and depression: state of the science. Hearth Lung. 2009;38(1):34-47.

10. Teles de Araújo, A. Relatórios do Observatório Nacional das Doenças Respiratórias de 2007 a 2009. Lisboa: Observatório Nacional das Doenças Respiratórias; 2010 [cited 2011 May 2]. Available from: http://www.ondr.org/relatorios_ondr.html. Portuguese

11. Lopez AD, Shibuya K, Rao C, Mathers CD, Hansell AL, Held LS, et al. Chronic obstructive pulmonary disease: current burden and future projections. Eur Respir J. 2006;27(2):397-412.

12. Murray $C$, Lopez AD. Alternative projections of mortality and disability by cause 1990-2020: Global Burden of Disease Study. Lancet. 1997;349(9064):1498-504.

13. Bárbara C, Rodrigues F, Dias H, Cardoso J, Almeida J, Matos MJ, et al. Prevalência da doença pulmonar obstrutiva crónica em Lisboa, Portugal: estudo Burden of Obstructive Lung Disease [Chronic obstructive pulmonary disease prevalence in Lisbon, Portugal: The Burden of Obstructive Lung Disease Study]. Rev Port Pneumol. 2013;19(3):96-105. Available from: http:///dx.doi. org/10.1016/j.rppneu.2012.11.004. Portuguese

14. Ramos-Cerqueira AT, Crepaldi AL. Qualidade de vida em doenças pulmonares crónicas: aspectos conceituais e metodológicos [Quality of life in chronic pulmonar diseases: conceptual and methodological aspects] J Pneumol. 2000;26(4):207-13. Portuguese

15. Ketelaars CA, Schlösser MA, Mostert R, Huyer Abu-Saad H, Halfens RJ, Wouters EF. Determinants of health-related quality of life in patients with chronic obstructive pulmonary disease. Thorax. 1996;51(1):39-43.

16. Dourado VZ, Antunes LC, Carvalho LR, Godoy I. Influência das características gerais na qualidade de vida de pacientes com doença pulmonar obstrutiva crónica [Influence of general clinical parameters on the quality of life of chronic obstructive pulmonary disease patient]. J Bras Pneumol. 2004;30(3):207-14. Portuguese

17. Curtis JR, Deyo RA, Hudson LD. Pulmonary rehabilitation in chronic respiratory insufficiency. 7: Health-related quality of life among patients with chronic obstructive pulmonar disease. Thorax. 1994;49(2):162-70.

18. Maurer J, Rebbapragada V, Borson S, Goldstein R, Kunik ME, Yohannes AM, et al. Anxiety and depression in COPD: current understanding, unanswered questions, and research needs. Chest. 2008;134(4 Suppl):43S-56S.

19. Ferrer M, Alonso J, Morera J, Marrades RM, Khalaf A, Aguar MC, et al. Chronic obstructive pulmonary disease stage and health-related quality of life. Ann Intern Med. 1997;127(12):1072-9.

20. Hajiro T, Nishimura K, Tsukino M, Ikeda A, Oga T, Izumi T. A comparison of the level of dyspnea vs disease severity in indicating the health-related quality of life of patients with COPD. Chest. 1999;116(6):1632-7.

21. Okubadejo AA, Jones PW, Wedzicha JA. Quality of life in patients with chronic obstructive pulmonary disease and severe hypoxemia. Thorax. 1996;51(1):44-7.
22. Tsukino M, Nishimura K, Ikeda A, Koyama H, Mishima M, Izumi T. Physiologic factors that determine the health-related quality of life in patients with COPD. Chest. 1996;110(4):896-903.

23. Renwick DS, Connolly MJ. Impact of obstructive airways disease on quality of life in older adults. Thorax. 1996;51(5):520-5.

24. Engström CP, Persson LO, Larsson S, Sullivan M. Health-related quality of life in COPD: why both disease-specific and generic measures should be used. Eur Respir J. 2001;18(1):69-76.

25. Sant'Anna CA, Stelmach R, Zanetti Feltrin MI, FilhoWJ, Chiba T, Cukier A. Evaluation of health-related quality of life in low-income patients with COPD receiving long-term oxygen therapy. Chest. 2003;123(1):136-41.

26. Hajiro T, Nishimura K, Tsukino M, Ikeda A, Oga T. Stages of disease severity and factors that affect the health status of patients with chronic obstructive pulmonary disease. Respir Med. 2000;94(9):841-6.

27. Di Marco F, Verga M, Reggente M, Maria Casanova F, Santus P, Blasi F, et al. Anxiety and depression in COPD patients: the roles of gender and disease severity. Respir Med. 2006;100(10):1767-74.

28. Stähl E, Lindberg A, Jansson SA, Rönmark E, Svensson K, Andersson F, et al. Health-related quality of life is related to COPD disease severity. Health Qual Life Outcomes. 2005;3:56.

29. Direção-Geral da Saúde. Orientações técnicas sobre diagnóstico e controlo da doença pulmonar obstrutiva crónica (DPOC): circular informativa $n^{\circ}$ 9/DSPCS, de 27/02/2007. Lisboa: DGS; 2007 [cited 2011 May 30]. Available from: http://www.dgs.pt. Portuguese

30. Esteves M, Viana RA. Avaliação da qualidade de vida relacionada com a saúde na doença pulmonar obstrutiva crónica. Rev Fac Ciênc Saúde. 2010;(7):466-75. Portuguese

31. Zigmond AS, Snaith RP. The Hospital Anxiety and Depression Scale.Acta Psychiatr Scand. 1983;67(6):361-70.

32. Pais-Ribeiro J, Silva I, Ferreira T, Martins A, Meneses R, Baltar M. Validation study of a Portuguese version of the Hospital Anxiety and Depression Scale. Psychol Health Med. 2007;12(2):225-37.

33. Cleland J, Lee AJ, Hall S. Associations of depression and anxiety with gender, age, health-related quality of life and symptoms in primary care COPD patients. Fam Pract. 2007;24(3):217-23.

34. NgTP, Niti M, Tan WC, Cao Z, Ong KC, Eng P. Depressive symptoms and chronic obstructive pulmonary disease: effect on mortality, hospital readmission, symptom burden, functional status, and quality of life. Arch Intern Med. 2007;167(1):60-7.

35. Snaith RP.The Hospital Anxiety and Depression Scale. Health Qual Life Outcomes. 2003;1:29.

36. Direção-Geral da Saúde. Orientações técnicas sobre reabilitação respiratória na doença pulmonar obstrutiva crónica (DPOC): circular informativa $n^{\circ}$ 40/DSPCD, de 27/10/2009. Lisboa: DGS; 2009 [cited 2011 May 30]. Available from: http://www.dgs.pt/?cr=14938. Portuguese

37. Jones PW, Quirk FH, Baveystock CM, Littlejohns P. A self-complete measure of health status for chronic airflow limitation: the St. George's Respiratory Questionnaire. Am Rev Respir Dis. 1992;145(6):1321-7.

38. Jones PW, Forde Y. St George's Respiratory Questionnaire: manual, version 2.2. London: St. George's University of London; 2009 [cited 2011 May 3]. Available from:http://www.healthstatus.sgul.ac.uk/SGRQ_download/SGRQ\%20 Manual\%20June\%202009.pdf

39. Souza TC, Jardim JR, Jones P.Validação do questionário do Hospital Saint George na doença respiratória (SGRQ) em pacientes portadores de doença pulmonar obstrutiva crônica no Brasil [Validation of the Saint George's Respiratory Questionnaire in patients with chronic obstructive pulmonar disease in 
Brasil]. J Pneumol. 2000;26(3):119-25. Portuguese

40. Meguro M, Barley EA, Spencer S, Jones PW. Development and validation of an improved, COPD-specific version of the St. George Respiratory Questionnaire. Chest. 2007;132(2):456-63.

41. Jones PW, Forde Y. St George's Respiratory Questionnaire for COPD Patients (SGRQ-C): manual, version 1.1. London: St. George's University of London; 2008 [cited 2011 May 20]. Available from: http://www.healthstatus. sgul.ac.uk/SGRQ_download/SGRQ-C\%20Manual\%202008.pdf

42. Ferrer M, Villasante C, Alonso J, Sobradillo V, Gabriel R, Vilagut G, et al. Interpretation of quality of life scores from the St. George's Respiratory Questionnaire. Eur Respir J. 2002;19(3):405-13.

43. Kovelis D, Segretti NO, Probst VS, Lareau SC, Brunetto AF, Pitta F. Validação do Modified Pulmonary Functional Status and Dyspnea Questionnaire e da escala do Medical Research Council para o uso em pacientes com doença pulmonar obstrutiva crônica no Brasil [Validation of the Modified Pulmonary Functional Status and Dyspnea Questionnaire and the Medical Research Council scale for use in Brazil patients with chronic obstructive pulmonary disease]. J Bras Pneumol. 2008;34(12):1008-18. Portuguese

44. Aarts $S$, van den Akker JM, Winkens B. The importance of effect sizes. Eur J Gen Pract. 2014;20(1):61-4.

45. Cohen J. Statistical power analysis for the behavioral sciences. 2nd ed. Hillsdale, NJ: Routledge; 1988. ISBN 9780805802832

46. Kline RB. Principles and practice of structural equations modeling. 2nd ed.
London: Guilford Press; 2005

47. Güder G, Brenner S, Angermann CE, Ertl G, Held M, Sachs AP, et al. GOLD or lower limit of normal definition? A comparison with expert-based diagnosis of chronic obstructive pulmonary disease in a prospective cohort-study. Respir Res. 2012;13(1):13.

48. DeTorres JP, Casanova C, Hernández C,Abreu J,Aguirre-JaimeA, Celli BR. Gender and COPD in patients attending a pulmonary clinic. Chest. 2005;128(4):2012-6.

49. Ferrari R, Tanni SE, Lucheta PA, Faganello MM, Amaral RA, Godoy I. Preditores do estado de saúde em pacientes com DPOC de acordo com o gênero [Gender differences in predictors of health status in patients with COPD]. J Bras Pneumol. 2010;36(1):37-43. Portuguese

\section{CONFLITO DE INTERESSES}

Os autores declaram não ter conflitos de interesses.

\author{
ENDEREÇO PARA CORRESPONDÊNCIA \\ Marlene Barros \\ Rua das Rosas, $n^{\circ 3}$ R/C, Palmeira 4700-737 Braga, Portugal \\ E-mail: anamarlene.39@gmail.com
}

Recebido em 25-10-2013

Aceite para publicação em 11-05-2014

\section{ABSTRACT}

\section{DETERMINANTS OF QUALITY OF LIFE IN PATIENTS WITH CHRONIC OBSTRUCTIVE PULMONARY DISEASE}

Objectives: To test the association between quality of life (QoL) and gender, age, body mass index, marital status, employment status, forced expiratory volume in 1 second, anxiety, depression and dyspnoea in patients with chronic obstructive pulmonary disease (COPD).

Type of study: Observational, cross-sectional study.

Location: Manuel Rocha Peixoto Family Health Unit and Gualtar Family Health Unit, Braga, Portugal.

Population: Patients over 40 years of age, diagnosed with COPD or chronic bronchitis, registered in the Gualtar and Manuel Rocha Peixoto Family Health Units.

Methods: Demographic data were collected and the Portuguese versions of the Hospital Anxiety and Depression Scale, the Medical Research Council Dyspnoea Questionnaire and the COPD-specific Saint George's Respiratory Questionnaire (SGRQ-C) were administered. The diagnosis of COPD was confirmed by spirometry, determining the forced expiratory volume in 1 second. A bivariate analysis of the study variables and QoL was performed. Statistically significant associations were tested by multiple linear regression.

Results: Sixty-five patients were included in the study. In the bivariate analysis, depression, anxiety and dyspnoea were related to all domains of the SGRQ-C. Gender and employment status also showed a significant bivariate correlation with "Activity", "Impact of disease" and the total SGRQ-C score. In the multiple linear regression analysis, gender and anxiety were not found to be significant predictors of outcomes. In the regression analysis, depression was a significant predictor for "Symptoms" and "Impact", employment status and dyspnoea were significant predictors for "Activity" and employment status, depression and dyspnoea were significant predictors for the SGRQ-C score.

Conclusion: Employment status, depression and dyspnoea should be considered in intervention strategies to improve QoL in patients with COPD.

Keywords: Chronic Obstructive Pulmonary Disease; Quality of Life. 